\author{
Leitner, Susanne
}

\title{
Netzwerkarbeit im Kinderschutz. Visionen für die Kooperation bei Gefährdung des Wohls von sonderpädagogisch markierten Kindern
}

Emotionale und soziale Entwicklung in der Pädagogik der Erziehungshilfe und bei Verhaltensstörungen : ESE 3 (2021) 3, S. 154-162

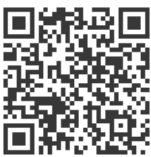

Quellenangabe/ Reference:

Leitner, Susanne: Netzwerkarbeit im Kinderschutz. Visionen für die Kooperation bei Gefährdung des Wohls von sonderpädagogisch markierten Kindern - In: Emotionale und soziale Entwicklung in der Pädagogik der Erziehungshilfe und bei Verhaltensstörungen : ESE 3 (2021) 3, S. 154-162 - URN: urn:nbn:de:0111-pedocs-226040 - DOI: 10.25656/01:22604

https://nbn-resolving.org/urn:nbn:de:0111-pedocs-226040

https://doi.org/10.25656/01:22604

in Kooperation mit / in cooperation with:

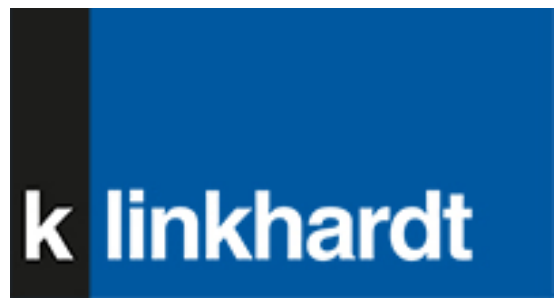

http://www.klinkhardt.de

\section{Nutzungsbedingungen}

Dieses Dokument steht unter folgender Creative Commons-Lizenz: http://creativecommons.org/licenses/by-nc-nd/4.0/deed.de - Sie dürfen das Werk bzw. den Inhalt unter folgenden Bedingungen vervielfältigen, verbreiten und öffentlich zugänglich machen: Sie müssen den Namen des Autors/Rechteinhabers in der von ihm festgelegten Weise nennen. Dieses Werk bzw. dieser Inhalt darf nicht für kommerzielle Zwecke verwendet werden und es darf nicht bearbeitet, abgewandelt oder in anderer Weise verändert werden.

Mit der Verwendung dieses Dokuments erkennen Sie die Nutzungsbedingungen an.

\section{Terms of use}

This document is published under following Creative Commons-License: http://creativecommons.org/licenses/by-nc-nd/4.0/deed.en - You may copy, distribute and transmit, adapt or exhibit the work in the public as long as you attribute the work in the manner specified by the author or licensor. You are not allowed to make commercial use of the work or its contents. You are not allowed to alter, transform, or change this work in any other way.

By using this particular document, you accept the above-stated conditions of use.

\section{Kontakt / Contact:}

\section{peDOCS}

DIPF | Leibniz-Institut für Bildungsforschung und Bildungsinformation Informationszentrum (IZ) Bildung

E-Mail: pedocs@dipf.de

Internet: www.pedocs.de

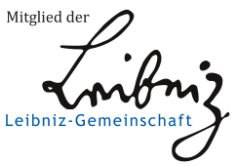



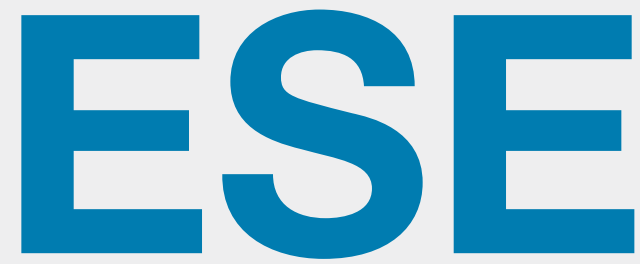

\section{Emotionale und Soziale}

Entwicklung in der Pädagogik der Erziehungshilfe und bei Verhaltensstörungen

\section{Heft 3}

Kompetent im NETZwerk:

Realität - Illusion - Vision?!

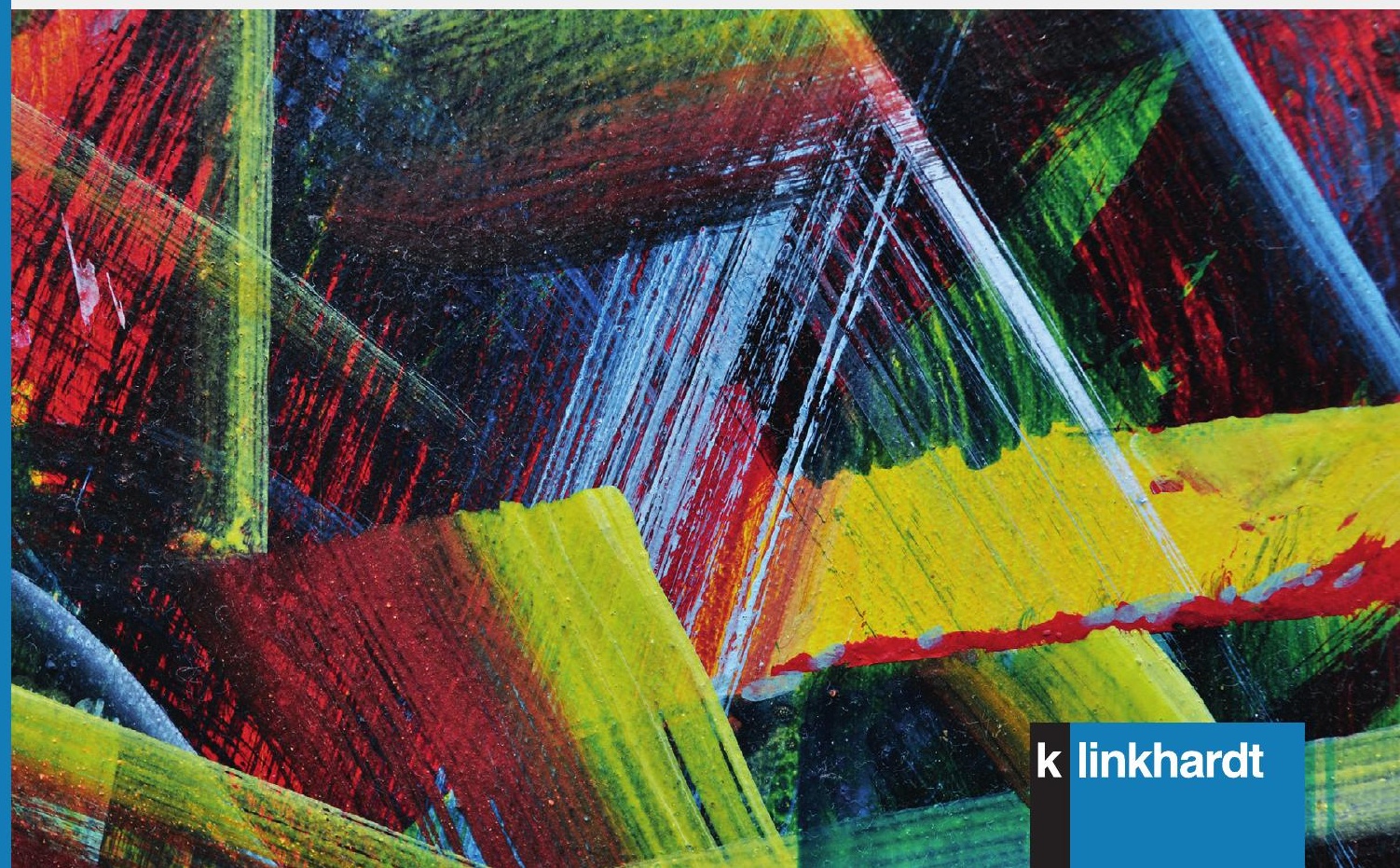




\title{
ESE
}

\author{
Emotionale und Soziale Entwicklung \\ in der Pädagogik der Erziehungshilfe und \\ bei Verhaltensstörungen
}




\section{ESE}

Emotionale und Soziale Entwicklung

in der Pädagogik der Erziehungshilfe und

bei Verhaltensstörungen

\section{Herausgebende Redaktion}

Stephan Gingelmaier (Sprecher der Redaktion)

Werner Bleher

Birgit Herz

Janet Langer

Lars Dietrich

Reinhard Markowetz

\section{Redaktionelle Unterstützung}

Anna Beyer 


\section{ESE}

Emotionale und Soziale Entwicklung in der Pädagogik der Erziehungshilfe und bei Verhaltensstörungen

3. Jahrgang (2021)

Heft 3

Kompetent im NETZwerk: Realität - Illusion - Vision?! 
Die Open Access-Publikation der Zeitschrift ESE wurde von verschiedenen Lehrstüblen und Personen der Dozierendenkonferenz der Forschenden und Lehrenden der „Pädagogik bei Verhaltensstörungen”। des Förderschwerpunkts „emotionale und soziale Entwicklung” finanziert.

Korrespondenzadresse:

Prof. Dr. Stephan Gingelmaier

Psychologie und Diagnostik im Förderschwerpunkt Emotionale und Soziale Entwicklung

PH Ludwigsburg

Reuteallee 46

71634 Ludwigsburg

Erscheinungsweise:

ESE Emotionale und Soziale Entwicklung in der Pädagogik der Erziehungshilfe und bei Verhaltensstörungen erscheint jährlich, jeweils im Sommer.

Die Hefte sind über den Buchhandel zu beziehen.

Das Einzelheft kostet EUR (D) 24,90, im Abonnement EUR (D) 24,90 (ggfs. zzgl. Versandkosten).

Das Abonnement für Studierende kostet EUR (D) 19,90 (ggfs. zzgl. Versandkosten)

nur bei Vorlage einer aktuellen Immatrikulationsbescheinigung.

Bestellungen und Abonnentenbetreuung:

Verlag Julius Klinkhardt

Ramsauer Weg 5

D-83670 Bad Heilbrunn

Tel: +49 (0)8046-9304

Fax: +49 (0)8046-9306

oder nutzen Sie unseren webshop:

www.klinkhardt.de

Bibliografische Information der Deutschen Nationalbibliothek

Die Deutsche Nationalbibliothek verzeichnet diese Publikation in der Deutschen Nationalbibliografie; detaillierte bibliografische Daten sind im Internet abrufbar über http://dnb.d-nb.de.

2021.1. () by Julius Klinkhardt.

Bildnachweis Umschlagseite 1: ( ) Petr Hrbek, 1992, Ohne Titel (Ausschnitt);

mit freundlicher Genehmigung von Ursula Binder und Martina Hoanzl.

Druck und Bindung: AZ Druck und Datentechnik, Kempten.

Printed in Germany 2021.

Gedruckt auf chlorfrei gebleichtem alterungsbeständigem Papier.

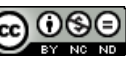

Die Publikation (mit Ausnahme aller Fotos, Grafiken und Abbildungen) ist veröffentlicht unter der Creative Commons-Lizenz: CC BY-NC-ND 4.0 International

https://creativecommons.org/licenses/by-nc-nd/4.0/

ISBN 978-3-7815-5903-5 Digital

doi.org/10.35468/5903

ISBN 978-3-7815-2467-5 Print

ISSN 2629-0170 


\section{Inhaltsverzeichnis}

Editorial der Herausgebenden 7

I Originalia

Perspektiven von Bediensteten des Jugendstrafvollzugs auf pädagogische Beziehung tiefenhermeneutische Einsichten aus einer qualitativ-empirischen Studie Janet Langer, Pierre-Carl Link, Ulrike Fickler-Stang und David Zimmermann

Erfassung der Lehrer*innen-Schüler*innen-Beziehung - ein Überblick über Erhebungsverfahren und -instrumente für die (sonder-)pädagogische Forschung und Praxis

Tatjana Leidig, Tijs Bolz, Émilie Niemeier, Jannik Nitz und Gino Casale

Multiprofessionalität und Netzwerke als Generallösung? -

Ein Plädoyer für einen Perspektivwechsel

Andrea Bethge

Mentalisieren im Netzwerk? Das Adaptive Mentalization-Based Integrative Treatment (AMBIT) als (inter-)professioneller Ansatz im Kontext der Pädagogik bei Verhaltensstörungen Andrea Dlugosch

Professionalität in pädagogischen Zwangskontexten:

Eine Annäherung aus der Perspektive der Pädagogik bei Verhaltensstörungen

Birgit Herz. .78

Professionalisierung im Kontext externalisierender Verhaltensprobleme -

Entwicklung eines Qualifizierungs- und Begleitkonzepts für Lehrkräfte an Förderschulen mit dem Förderschwerpunkt Emotionale und soziale Entwicklung

Tatjana Leidig, Charlotte Hanisch, Ulrike Vögele, Émilie Niemeier, Silke Gerlach

und Thomas Hennemann

Kunstunterricht im Kontext von Unterrichts- und Verhaltensstörungen -

Zur Konzeption eines didaktischen Planungsmodells für Kunst- und

Sonderpädagoginnen und -pädagogen

Daniel Ricci.....

Netzwerk B ${ }^{2}$ : Betrieb und Berufsschule in der inklusiven Ausbildungsvorbereitung eine Fragebogenstudie zu zentralen Bedingungs- und Wirkfaktoren

Francesco Ciociola, Stefanie Roos und Christoph de Oliveira Käppler.

aRT - ein Ansatz zur wissenschaftlich gestützten Schulberatung Janna Rühl, Pascal Schreier, Stephanie Blatz und Roland Stein 
Praxis-Forschungs-Netzwerke am Beispiel des Projekts „Geschwisterklassen“

Désirée Laubenstein und David Scheer

III Praxis und Theorie

Netzwerkarbeit im Kinderschutz. Visionen für die Kooperation bei Gefährdung des Wohls von sonderpädagogisch markierten Kindern

Susanne Leitner.

Umsetzung und Evaluation eines multiprofessionellen und systemübergreifenden Inklusionskonzeptes für Kinder und Jugendliche im Schulalter Karolina Urton, Sophia Hertel und Thomas Hennemann

IV Buchbesprechungen

Das eigensinnige Kind. Über unterdrückten Widerstand und die Formen ungelebten Lebens - ein gesellschaftspolitischer Essay

Pierre-Carl Link

V Forum: Kurzberichte aus den Bundesländern

Bayern: Neuer Standort der Pädagogik bei Verhaltensstörungen in Regensburg

Bernhard Rauh, Pierre-Carl Link und Philipp Abelein

VI Neues aus dem Fach (Zeitraum 2020 - Friuhjahrr 2021) 


\title{
Netzwerkarbeit im Kinderschutz. Visionen für die Kooperation bei Gefährdung des Wohls von sonderpädagogisch markierten Kindern ${ }^{1}$
}

\author{
Susanne Leitner
}

1 Im Sinne der UN-Kinderrechtskonvention sollen in diesem Artikel mit dem Begriff „Kinder“ alle Personen gemeint werden, die das 18. Lebensjahr noch nicht vollendet haben. Die Beschäftigung mit Kindeswohl(gefährdung) legt einerseits nahe, Kinder als Trägerinnen und Träger von spezifischen (Kinder-)Rechten zu betrachten - was trotz gelegentlich abweichender Handhabung in der Praxis auch noch auf Jugendliche zutrifft.

Mit der Formulierung ,sonderpädagogisch markiert" schließe ich an rassismuskritische Diskurse an und möchte darauf aufmerksam machen, dass die Zuschreibung des sonderpädagogischen Förderbedarfs an dieser Stelle als sozial und institutionell hervorgebrachte Kategorie gelesen wird, die zunächst eine Information über die Positioniertheit des Kindes, nicht aber über sein bzw. ihr Sosein beinhaltet. 


\section{Abstract}

Der Beitrag reflektiert die Notwendigkeit, Wissen über Rechtsgrundlagen und Verfahrensabläufe bei Verdacht auf Kindeswohlgefährdung in Schulen zu implementieren und die Kooperation zwischen Schule und Jugendhilfe zu stärken. Anhand zweier im interdisziplinären Dia$\log$ gewonnener Fallbeispiele werden Impulse vorgeschlagen, wie das gegenseitige Verständnis von unterschiedlichen Institutionslogiken verbessert und der Aspekt des Kinderschutzes in der Pädagogik im Förderschwerpunkt ESE verankert werden könnte.

\section{Keywords}

Kinderschutz, Kindeswohlgefährdung, interdisziplinäre Kooperation 


\section{Einführung}

Dieser Beitrag entsteht aufgrund mehrerer Begebenheiten, die mich zum Staunen darüber gebracht haben, wie wenig Beachtung das Thema Kinderschutz, insbesondere die dabei gesetzlich vorgesehene multiprofessionelle Netzwerkarbeit, in Bildungsprozessen von Sonderpädagoginnen und Sonderpädagogen findet. Mit „Staunen“ verwende ich bewusst einen nichtwissenschaftlichen Begriff und sehe diesen Beitrag eher als Beginn und nicht als Ergebnis eines Reflexionsprozesses. Zu den genannten Begebenheiten zählte neben Gesprächen mit Studierenden und Inklusionslehrerinnen und -lehrern insbesondere eine studentische Qualifikationsarbeit zum Thema Kindeswohlgefährdung, die auf Interviews mit Jugendamtsmitarbeiterinnen und -mitarbeitern basierte und in der das Bundeskinderschutzgesetz mit seiner Aufforderung zur Netzwerkarbeit mit keinem einzigen Wort Erwähnung fand. Diese Irritation möchte ich als Chance nutzen, dafür zu plädieren, Kooperation im Kinderschutz als Kernaufgabe von Schule zu begreifen - insbesondere auch für Lehrerinnen und Lehrer ${ }^{2}$ im sogenannten „Förderschwerpunkt ESE“, die verstärkt mit Kindern in riskanten Lebenslagen konfrontiert (Herz \& Zimmermann, 2018) und auch als Expertinnen und Experten für diese Kinder adressiert werden.

\section{Kindeswohl und Kindeswohlgefährdung}

Eine differenzierte fachliche und juristische Auseinandersetzung mit den Begriffen Kindeswohl und Kindeswohlgefährdung kann hier aus Platzgründen nicht erfolgen. Das Kindeswohl ist ein unbestimmter Rechtsbegriff, der stets für den Einzelfall definiert und an den Bedürfnissen von Kindern (Brazelton \& Greenspan, 2002) ausgerichtet werden muss (Günderoth, 2017). Folgerichtig können auch Gefährdungen des Kindeswohls viele Facetten haben. Kinderschutz kann daher als Arbeitsfeld mit besonders hoher Komplexität und Mehrdeutigkeit beschrieben werden (Koch, Pineiro \& Pasche, 2019).

Als zentrale, aber nicht abschließende Kategorien werden zumeist Formen von Vernachlässigung (z.B. materieller oder emotionaler Art und Verletzung der Aufsicht und Sorge) sowie körperlicher, seelischer und sexualisierter Gewalt beschrieben (Günderoth, 2017; Bathke, Bücken \& Fiegenbaum, 2019). Liegen sogenannte gewichtige Anhaltspunkte für eine Gefährdung vor, nimmt der Staat, vertreten durch ein Jugendamt, sein Wächteramt wahr, um die Gefahr einzuschätzen und abzuwenden (Günderoth, 2017; Bathkeet et al., 2019). Dies geschieht insbesondere auf Grundlage des \$8a SGB VIII, der das Vorgehen von Jugendhilfeträgern regelt. Betont werden soll hier die Bedeutung des insbesondere für Lehrerinnen und Lehrer relevanten Bundeskinderschutzgesetztes (BuKiSchG), das im Jahr 2012 in Kraft getreten ist. Dieses fungiert als Rahmengesetz, in dem u.a. das Gesetz zur Kooperation und Information im Kinderschutz (KKG) enthalten ist. Das KKG regelt in $\$ 4$ die Beratung und Informationsweitergabe durch Berufsgeheimnisträgerinnen und -träger (also auch Lehrkräfte), um die interdisziplinäre Kooperation im Kinderschutz zu verbessern (Bertsch, 2016; $\$ 4$ $\mathrm{KKG}$ ). Von zentraler Bedeutung ist insbesondere das Recht auf Beratung durch eine „insoweit erfahrene Fachkraft“ nach $\$ 8$ a SGB VIII und die relativ klare Regelung des Vorgehens im Verdachtsfall (s. Abb. 1).

2 Im Folgenden wird von Lehrerinnen und Lehrern die Rede sein, da das Thema für alle Schulformen relevant ist. Wie oben beschrieben sehe ich aber für Sonder- oder Inklusionslehrerinnen und -lehrer, die im Förderschwerpunkt Emotionale und Soziale Entwicklung tätig sind, besondere Dringlichkeit. 


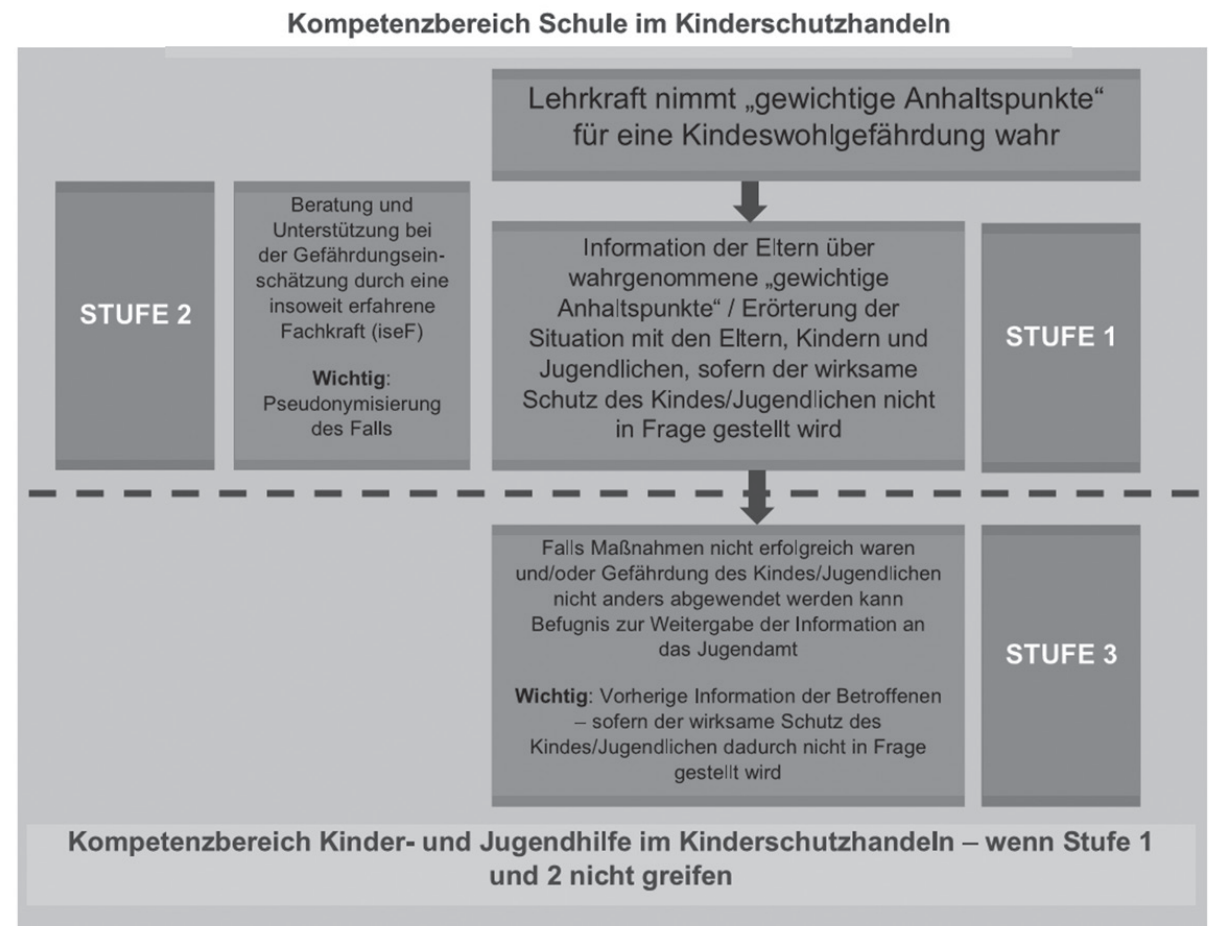

Abb. 1: Regelung des Vorgehens im Verdachtsfall (Bathke et al., 2019, S. 35)

Kooperation mit insoweit erfahrenen Fachkräften und auch mit Eltern ist im Verdachtsfall also nicht nur eine sinnvolle Option, sondern gesetzlich vorgeschrieben. In einer 2016 durchgeführten bundesweiten Befragung zeigte sich jedoch, dass die meisten Schulen nicht ausreichend über das BuKiSchG und seine Umsetzung informiert waren (Zimmermann, 2016). Ob sich dies in den letzten Jahren wesentlich geändert hat, kann bezweifelt werden (Zimmermann, 2020).

Wie interdisziplinäre Kooperation gelingen kann, wurde für verschiedene Handlungsfelder wie auch den Kinderschutz in der vorliegenden Fachliteratur bereits diskutiert (Bertsch, 2016; Zimmermann, 2016). Zu nennen sind insbesondere Implementierung fallunabhängiger Kooperationsformen wie regelmäßige gemeinsame Fachtage, Fortbildungen und Arbeitskreise, in denen sich Akteurinnen und Akteure aus Schule und Jugendhilfe kennen lernen, über ihre jeweiligen Handlungslogiken verständigen und gegenseitig über Neuerungen wie Gesetzesnovellen oder lokale und regionale Initiativen auf dem Laufenden halten können (Bertsch, 2016; Zimmermann, 2016).

Es zeigt sich, dass Kooperation im Krisenfall dann besser funktioniert, wenn es auch fallunabhängige Kooperation gibt, die geeignet ist, Vertrauen zwischen Kooperationspartnern ohne unmittelbaren Handlungsdruck aufzubauen. Diese Kooperation ermöglicht es, die anderen an der Kooperation Beteiligten kennenzulernen und ihre Handlungslogiken zu verstehen (Bertsch, 2016, S. S. 43f.). 


\section{Interdisziplinäre Praxisreflexion}

Die folgenden Praxisreflexionen basieren auf dem engen Austausch mit einer KinderschutzExpertin aus der Jugendhilfe (im Folgenden: Frau K.) und setzen insoweit den Gedanken interdisziplinärer Zusammenarbeit um.

Frau K. ist als Sozialarbeiterin und insoweit erfahrene Fachkraft im Kinderschutz tätig. Sie hat langjährige Erfahrungen auf verschiedenen Funktionsebenen in unterschiedlichen Handlungsfeldern der Jugendhilfe gesammelt. Auf ihren Wunsch verzichte ich hier darauf, ihren Namen zu nennen. Bei unseren Gesprächen, die wir in Anlehnung an das Ero-Epische Gespräch nach Girtler (1996) durchgeführt haben, ging es uns um das gemeinsame dialogische Hervorbringen und Greifbarmachen impliziten Wissens (Bohnsack, 2013). Dabei knüpften Frau K. und ich bei der Vorarbeit zu diesem Beitrag an unsere bestehende freundschaftliche Alltagspraxis an, über fachliche Themen, aus unseren jeweiligen Berufskontexten zu reflektieren, nahmen diese Gespräche aber nun auf Tonband auf. Später hörten wir die Aufnahmen wieder an und identifizierten die Aspekte, die uns am relevantesten erschienen. Dies geschah also nicht im Sinne einer regelgeleiteten wissenschaftlichen Auswertung, sodass das Ergebnis eben auch als interdisziplinäre Praxisreflexion und nicht als qualitative Forschung anzusehen ist. An der finalen Texterstellung waren wir beide beteiligt.

Der erste Impuls, den wir aus diesem Prozess gewannen, war, dass wir die oben genannten, bereits umfangreich in der Literatur beschriebenen Formen fallunabhängiger Kooperation ebenfalls für zentral halten und hervorheben möchten. Dazu gehört immanent, dass die regional geltenden institutionsübergreifenden Absprachen über Vorgehensweisen im Verdachtsfall stetig aktualisiert und an alle Kolleginnen und Kollegen in Jugendamt und Schule transportiert werden.

Darüber hinaus haben wir drei Impulse herausgearbeitet, die wir mit dem Fokus auf die Bildung angehender Lehrerinnen und Lehrer im Bereich ESE als hilfreich diskutieren wollen.

\section{Impuls 1: (Inklusions-) Lehrkräfte brauchen mehr Gesprächsführungskompetenz, insbe-} sondere im Formulieren existentieller und unangenehmer Themen.

Das nachfolgende Fallbeispiel hat so im Rahmen der Frühen Bildung stattgefunden - wäre aber in ähnlicher Form durchaus auch im Schulkontext denkbar.

Die Meldung einer Kita bezüglich eines Kindes aus einer multipel belasteten Familie wird von einem Jugendamt als kinderschutzrelevanter Fall eingeschätzt. Aufgrund gut funktionierender Abläufe gelingt es innerhalb kurzer Zeit, eine Sozialpädagogische Familienhilfe mit einer hohen Stundenzahl zu installieren. Die Hilfe wird von der Familie gut angenommen; insbesondere die Mutter öffnet sich sehr schnell und ist bereit, über ihre massive und komplexe Überforderung sowie die von ihrem Ehemann an ihr und den Kindern ausgeübte Gewalt zu sprechen um gemeinsam nach einer Lösung zu suchen. Damit die Probleme noch nicht aus der Welt geschaff, aber bearbeitbar geworden. Leider ist diese Falldarstellung erst eine Erfolgsgeschichte im zweiten Anlauf, da es durch die Verkettung verschiedener Umstände mehrere Monate gedauert hat, bis der Fall vom Jugendamt als kinderschutzrelevant eingeschätzt werden konnte. Dass es in der Familie Probleme hinsichtlich der Erziehung gibt, ist in der Kita schon seit Langem aufgefallen. Das älteste Kind zeigt so massive Verhaltensschwierigkeiten, dass der Besuch der Kita ganz beendet wird. Weitere Hilfe wird nicht hinzugezogen. Auch in der Arbeit mit einem der jüngeren Kinder nehmen die Erzieherinnen und Erzieher Warnsignale wahr, die sie intern notieren, aber nicht weiterverfolgen. Elterngespräche thematisieren stattdessen Nebenschauplätze wie das ungesunde Frühstück, das das 
Kind mitbringt - eine Mahnung, die wiederum von der Mutter nicht als dringlich dechiffriert wird. Dass ernsthafte Sorgen im Raum stehen, die dringend zur Sprache gebracht und bearbeitet werden müssen, wird nicht thematisiert. Da die Erzieherinnen und Erzieher weder manifeste Beweise haben noch ihre latenten Sorgen aussprechen, bleibt das Dahinterliegende unnötig lange im Verborgenen, bevor letztendlich eine Meldung ans Jugendamt gegeben wird.

Durch die Thematisierung des ungesunden Frühstücks wird im Elterngespräch ein existentielles Thema gestreift - ungesunde oder Mangelernährung kann ab einem gewissen Ausmaß ebenfalls als Vernachlässigung und (im Extremfall lebensbedrohliche) Kindeswohlgefährdung verstanden werden. Das Thema der Bedrohung scheint also symbolisch im Raum zu stehen. Die eigentliche, sich nachträglich bestätigende Befürchtung aber, dass die Kinder in ihrer Familie Gewalt ausgesetzt sein könnten, wird durch die Erzieherinnen und Erzieher nicht formuliert. Nach geltendem Recht hätten sie sich, wenn sie die Tragweite erkannt hätte, von einer insofern erfahrenen Fachkraft beraten lassen, die Eltern über ihre Sorge, dass Anhaltspunkte zur Kindeswohlgefährdung vorliegen, informieren und sie in die Gefährdungseinschätzung mit einbeziehen müssen. Im Rahmen dieser Gespräche hätten zu einem frühen Zeitpunkt bereits Vorschläge für niederschwellige Hilfen (z.B. Frühe Hilfen, Beratungsstellen usw.) gemacht werden können. Wenn diese sich als nicht ausreichend zur Abwendung der Gefährdung herausgestellt hätten, wäre eine Meldung, notwendig geworden (\$8a Abs. 4 SGB VIII).

Die Sozialarbeiterinnen und Sozialarbeiter der Familienhilfe sind erstaunt, nach welch kurzer Zeit die Mutter bereit ist, über den sich bestätigenden Verdacht der häuslichen Gewalt zu sprechen. Sie zeigt sich offen, über das Thema zu sprechen und leugnet das Problem nicht. Vielmehr drückt sie Wertschätzung und Dankbarkeit für die Hilfestellung aus. Diese Offenheit könnte nicht zuletzt darauf zurückzuführen sein, dass der Verdacht im Jugendamt nicht beschönigt, sondern klar formuliert und als Sorge, das Wohl der Kinder könnte dadurch gefährdet werden, benannt wurde. Das klare Benennen schätzen wir als maßgeblich dafür ein, dass es der Mutter möglich wurde, die Gefährdung selbst offen zu legen und damit der Bearbeitung zugänglich zu machen.

Wenn wir dieses Schlagen nicht so klargemacht hätten, wäre sie [die Mutter, SL] auch nicht drauf gekommen, das aufzumachen, glaube ich. Weil das klar war, das war ein Thema, was wir wirklich auch als Gefährdung für die Kinder und auch für sie betrachtet haben. Da ist ganz viel, was über diesen Rand// das hat sie vorher in den präventiven Hilfen nicht erzählt, obwohl sie sie angenommen hat. (Gespräch 2, 23:32)

$\mathrm{Zu}$ diesem leider unnötig zeitverzögerten Erfolg beigetragen hat, dass die Jugendamtsmitarbeitnden der Mutter in für sie gut verständlicher Weise „[...] die Bedeutung klargemacht haben, was das heißt, weil wir schon auch gesagt haben, dass das den Kindern schadet" (Gespräch 2; 24:21) ohne dabei in Moralisierungen, Drohungen oder Verfolgungszenarien zu verfallen. So konnte thematisiert werden, „(...) was Gewalt macht, warum das nicht OK ist, auch wenn es viele machen, und warum es auch nicht darum geht, sie zu verurteilen, sondern dass es da Änderungen braucht" (Gespräch 2, 25:24).

Beratung und Elternarbeit gehören zwar zur (sonderpädagogischen) Lehrerbildung, jedoch - in den mir bekannten Zusammenhängen - eher selten in einem Umfang, der zur Gesprächsführung zu so heiklen Themen befähigen würde. Wünschenswert wäre eine höhere Priorisierung dieses Kompetenzbereichs, unbedingt verbunden mit der Auseinandersetzung 
mit eigenen Ängsten, die zur Vermeidung von Konfrontation mit solchen Themen führen können (Gerspach, 2020).

Impuls 2: Lehrerinnen und Lehrer sollten lernen, wie eine "gute" Meldung geht (aber auch „schlechte“ Meldungen sollten von Jugendämtern als Beginn eines fehlerfreundlichen Dialoges gesehen und verbreitet werden)

Fallbeispiel: Von einer Schule geht eine Meldung des Verdachts auf Kindeswohlgefährdung an ein Jugendamt. Die Meldung besteht aus einer langen Liste von außerordentlich gravierenden Gefährdungen, die zu sofortigen und einschneidenden Maßnahmen führen müssten. Allerdings stellen die Jugendamtsmitarbeitenden bei Kontaktaufnahme mit den Eltern schnell fest, dass keine der von der Schule behaupteten Tatsachen verifizierbar ist und keine gewichtigen Anhaltspunkte gefunden werden können. Da das im Teenageralter befindliche Kind keine freiwillige Hilfe annimmt und sich selbst auch nicht als gefährdet wahrnimmt, wird der Fall nicht mehr als kinderschutzrelevant eingestuft. Eine weitere Kommunikation zwischen Jugendamt und Schule findet nicht statt. Dass Akteurinnen und Akteure beider Institutionen verärgert und zur weiteren Kooperation wenig motiviert sind, ist wahrscheinlich.

$\mathrm{Zu}$ diesem unproduktiven Ergebnis geführt hat wohl, dass die meldende Lehrperson bzw. Schulleitung unhaltbare Behauptungen aufgestellt hatte, anstatt aufgrund von Beobachtungen ihre vermutlich begründete Sorge zu formulieren.

Das Jugendamt wiederum verfolgte nicht nachdrücklich genug das Ziel, mit der Lehrperson in den Dialog zu treten, die der Meldung zugrundeliegenden Beobachtungen und Überlegungen zu erfragen und sie gegebenenfalls bei der Formulierung ihrer Sorge sowie eventuell vorhandener gewichtiger Anhaltspunkte zu unterstützen. So hätte eruiert werden können, dass die betreffende Schülerin mit einiger Wahrscheinlichkeit in Zukunft den benannten Gefahren ausgesetzt sein könnte. Dabei hätten beide Institutionen über niederschwellige Unterstützungsmöglichkeiten nachdenken können. Voraussetzung wäre gewesen, dass sowohl Jugendamt als auch Schule als handlungsleitend die gegenseitige Annahme von Fachwissen und Sorge zum Wohl und zur Förderung des Kindes - trotz institutionslogischer Unterschiede in der Ausdrucksweise voraussetzen.

Irgendwo gibt es einen Grund, warum die [Lehrerinnen und Lehrer, SL] auf die Idee gekommen sind. (...) Eigentlich müsste ein Gespräch stattfinden, wie kommen Sie auf die Punkte? Weil da sind sicher Übertragungen, Ängste, auch irgendwas zu stark wahrgenommen, aber nicht ohne Grund. Die spüren ja was. Die kommen ja nicht ohne Grund auf diese ganzen Themen (Gespräch 1, 18:12).

Wenn auch nicht alle Lehrerinnen und Lehrer genau Bescheid wissen müssen, wie gewichtige Anhaltspunkte zu formulieren sind, damit sie nach $\$ 8$ a SGB VIII vom Jugendamt bearbeitbar werden, sollte doch wenigstens die Hinzuziehung einer insoweit erfahrenen Fachkraft, die hierbei beraten kann, bekannt und angebahnt sein. Gleichermaßen sollten sich Jugendamtsmitarbeitende die Zeit nehmen, auch Meldungen, die nicht die erwartete Qualität aufweisen, einer Sinnhaftigkeitsunterstellung zu unterziehen und die hinter ungelenk formulierten Meldungen liegenden Sorgen, die sich Lehrerinnen und Lehrer um Kinder machen, zu würdigen. Hilfreich könnte dabei auch sein, wenn sich Lehrerinnen und Lehrer das in der Jugendhilfe häufig verwendete Wort „Sorge“ mehr als Denkfigur und Terminus ihrer eigenen Profession aneignen würden. 


\section{Impuls 3: „Sorge“ als Konzept und Terminus auch von Lehrerinnen und Lehrern}

Als letzten Impuls schlagen wir vor, dass bei jedem Sonderpädagogischen Feststellungsverfahren, zumindest, wenn es hinsichtlich Auffälligkeiten im Bereich der emotional-sozialen Entwicklung durchgeführt wird, standardmäßig die Frage stellen, inwiefern der Anlass für diese Diagnostik mit einer Kindeswohlgefährdung in Verbindung stehen könnte - unabhängig davon, in wie vielen Fällen letztlich keine derartigen Anhaltspunkte gefunden werden. In den Fällen, in denen Anhaltspunkte aber nicht von vorneherein mit ausreichender Sicherheit ausgeschlossen werden können, könnte die Einbeziehung einer insoweit erfahrenen Fachkraft und Durchführung eines Sechsaugengesprächs, wie es im Verdachtsfall üblich ist (die Vorgänge sind jeweils lokal oder regional mit den Jugendämtern geregelt und können leicht variieren) zur Routine werde. Diese Routine würde bereits zur Stabilisierung der Zusammenarbeit beitragen.

Dieses Vorgehen halte ich für angezeigt, da bei der Einschätzung der gewichtigen Anhaltspunkte für eine akute oder latente Kindeswohlgefährdung stets die Frage gestellt werden muss, ob eine erhebliche Schädigung der Entwicklung zu befürchten ist (Günderoth, 2017). Wenn Kinder innere Themen durch Verhaltensweisen zum Ausdruck bringen, die in ihrer Vehemenz zum Ausschluss aus dem System der allgemeinen Schule führen können, kann dies durchaus als erhebliche Schädigung der Entwicklung diskutiert werden.

Meldungen über den Verdacht auf Kindeswohlgefährdung werden aus Sorge, dass Kinder in einer Notsituation sein könnten, getätigt. Sonderpädagogische Feststellungsdiagnostik jedoch wird, so behaupte ich, nicht selten dann veranlasst, wenn durch Verhalten von Schülerinnen und Schülern ihre Lehrerinnen und Lehrer in Not geraten. Sicherlich geht es diesen dabei immer auch um das Wohl des Kindes. Vielleicht müsste jedoch die Logik der Sorge von der Sonderpädagogik theoretisch und konzeptionell stärker verankert und in der Lehrerbildung vermehrt als solche formuliert werden. Dies würde auch implizieren, dass Verhalten von Kindern, das sonderpädagogische Feststellungsdiagnostik veranlasst, wieder verstärkt auf die dahinterliegenden szenischen Botschaften befragt wird (Rauh, 2010; Gerspach, 2020). Inklusionslehrerinnen und -lehrer im Förderschwerpunkt ESE könnten sich nicht vorrangig als Expertinnen und Experten für Schülerinnen und Schüler mit Verhaltensstörungen verstehen, sondern in erster Linie als Expertinnen und Experten für Schülerinnen und Schüler in (emotionaler oder sozialer) Not.

\section{Fazit}

Abschließend kann festgehalten werden, dass die pädagogische Praxis viele Anlässe zu Austausch, Kooperation und Vernetzung bietet, die sowohl von Schule als auch von Jugendhilfe diskursiv genutzt werden können und sollten. Ein Ziel könnte dabei sein, eine gemeinsame Sprache zu finden, die es erlaubt, von- und miteinander zu lernen mit dem Ziel „(...) dass man zu einer inhaltlichen Kooperation, die Fehler erlaubt, kommen muss" (Gespräch 1, 30:54). Geltendes Recht verlangt, dass Kinderschutz zu einem immanenten Teil des Professionsverständnisses von Lehrerinnen und Lehrern wird. 


\section{Literatur}

Bathke, S., Bücken, M, Fiegenbaum, D. (2019). Praxisbuch Kinderschutz interdisziplinär. Wie die Kooperation von Schule und Jugendhilfe gelingen kann. Wiesbaden: Springer.

Bertsch, B. (2016). Wirkungen des Bundeskinderschutzgesetzes - wissenschaftliche Grundlagen. Ergebnisbericht zu Erhebungen im Gesundheitswesen. https://www.dji.de/fileadmin/user_upload/bibs2016/BKiSchG_Gesundheitswesen. pdf. Zugegriffen am 23. November 2020.

Brazelton, T. B. \& Greenspan, S. I. (2002). Die sieben Grundbedürfnisse von Kindern. Was jedes Kind braucht, um gesund aufzuwachsen, gut zu lernen und glücklich zu sein. Weinheim und Basel: Beltz Juventa.

Bohnsack, R. (2013). Dokumentarische Methode und Logik der Praxis. In A. Lenger et al. (Hrsg.), Pierre Bourdieus Konzeption des Habitus (S. 175-200). Wiesbaden: Springer

Koch, M., Pineiro, E. \& Pasche, N. (2019). „Wir sind ein Dienst, keine Behörde.“ Multiple institutionelle Logiken in einem Schweizer Jugendamt - ein ethnografisches Fallbeispiel aus der street-level bureaucracy. Forum Qualitative Sozialforschung/Forum: Qualitative Social Research, 20(2). https:/www.ssoar.info/ssoar/handle/document/62798.

Gerspach, M. (2020): Zum Verständnis von Gruppe und institutioneller Abwehr in der Psychoanalytischen Pädagogik. Gruppenanalyse: Zeitschrift für gruppenanalytische Psychotherapie, Beratung und Supervision 30 (1), 12-36.

Gesetz zur Kooperation und Information im Kinderschutz. https://www.gesetze-im-internet.de/kkg/. Zugegriffen: 23.11.2020.

Gesetz zur Stärkung eines aktiven Schutzes von Kindern und Jugendlichen. (Bundeskinderschutzgesetz-BKiSchG) vom 22. Dezember 2011. https://dejure.org/BGBl/2011/BGBl._I_S._2975 Zugegriffen: 23.11.2020

Girtler, R. (1996). Die 10 Gebote der Feldforschung. Sozialwissenschaften und Berufspraxis, 19(4), 378-379.

Günderoth, M. (2017). Kindeswohlgefährdung. Die Umsetzung des Schutzauftrages in der verbandlichen Jugendarbeit. Gießen: Psychosozial.

Herz, B. \& Zimmermann, D. (2018). Beziehung statt Erziehung? Psychoanalytische Perspektiven auf pädagogische Herausforderungen in der Praxis mit emotional-sozial belasteten Heranwachsenden. In R. Stein \& T. Müller (Hrsg.), Inklusion im Förderschwerpunkt emotionale und soziale Entwicklung (S.150-177). 2., erweiterte und überarbeitete Auflage, Stuttgart: Kohlhammer.

Rauh, Bernhard (2010): Szenisches Verstehen. In B. Ahrbeck \& M. Willmann (Hrsg.), Pädagogik bei Verhaltensstörungen. Ein Handbuch (S. 173-181). Stuttgart: Kohlhammer,

Sozialgesetzbuch (SGB) - Achtes Buch (VIII) - Kinder- und Jugendhilfe - (Artikel 1 des Gesetzes v. 26. Juni 1990, BGBl. I S. 1163. https://www.gesetze-im-internet.de/sgb_8/_8a.html. Zugegriffen: 22.11.2020

Übereinkommen über die Rechte des Kindes. Resolution 44/25 der Generalversammlung der Vereinten Nationen vom 20.November 1989. https://www.kinderrechtskonvention.info/uebereinkommen-ueber-die-rechte-deskindes-370/. Zugegriffen am 01. Dezember 2020.

Zimmermann, J. (2016). Das Bundeskinderschutzgesetz aus Schulperspektive Ergebnisse einer bundesweiten Erhebung an Schulen. Sozial Extra 40(5), 42-45 